\title{
Contribution à un dialogue
}

Réponse des auteurs de l'ouvrage recensé

Yves Reuter, Bertrand Daunay, Isabelle Delcambre, Cora Cohen-Azria et Dominique Lahanier-Reuter

\section{(2) OpenEdition}

\section{Journals}

Édition électronique

URL : http://journals.openedition.org/educationdidactique/1068

DOI : 10.4000/educationdidactique. 1068

ISSN : 2111-4838

\section{Éditeur}

Presses universitaires de Rennes

\section{Édition imprimée}

Date de publication : 30 mai 2011

Pagination : 121-126

ISBN : 978-2-7535-1449-2

ISSN : 1956-3485

\section{Référence électronique}

Yves Reuter, Bertrand Daunay, Isabelle Delcambre, Cora Cohen-Azria et Dominique Lahanier-Reuter, "Contribution à un dialogue », Éducation et didactique [En ligne], 5-1 | 2011, mis en ligne le 30 mai

2011, consulté le 10 décembre 2020. URL : http://journals.openedition.org/educationdidactique/1068 ; DOI : https://doi.org/10.4000/educationdidactique.1068

Ce document a été généré automatiquement le 10 décembre 2020.

Tous droits réservés 


\title{
Contribution à un dialogue
}

\author{
Réponse des auteurs de l'ouvrage recensé \\ Yves Reuter, Bertrand Daunay, Isabelle Delcambre, Cora Cohen-Azria et \\ Dominique Lahanier-Reuter
}

\section{RÉFÉRENCE}

Yves Reuter et al., 2010, Dictionnaire des concepts fondamentaux des didactiques, Paris, De boeck.

1 Nous souhaitons tout d'abord remercier la revue Éducation et didactique. Il nous semble en effet que choisir notre ouvrage comme support du premier compte rendu sous forme de débat manifeste, au-delà des critiques légitimes dont il peut faire l'objet, sa reconnaissance comme outil permettant de susciter la discussion entre les diverses didactiques, de penser leurs évolutions et, nous l'espérons, de contribuer à «la nécessité d'instaurer un champ didactique rassembleur » (C. Orange).

2 Nous considérons de surcroît que cette discussion critique, à l'occasion de la parution plus rapide que prévue de la seconde édition du Dictionnaire en raison du succès de la première édition, prolonge les comptes rendus effectués par des didacticiens de différentes disciplines (J-P. Astolfi, J.L. Dumortier, J. Lebeaume, H. Romian, N. TutiauxGuillon notamment...) dans des revues françaises ou étrangères ainsi que les échanges que nous avons pu avoir avec d'autres chercheurs, des formateurs ou des étudiants. Au vu de ces comptes rendus et de ces échanges, il nous semble que la réception de cet ouvrage a été assez nettement favorable pour quelques raisons principales :

- il comble un manque, réunissant de manière accessible concepts et informations (notamment bibliographiques) issus des différentes didactiques qui ont, trop souvent encore, tendance à vivre en champ clos (voir les dictionnaires ou glossaires propres à telle ou telle didactique, voire à tel ou tel courant) ;

- il permet aux didactiques de communiquer entre elles dans la mesure où sa conception est " ouverte » : n'excluant aucune didactique, aucun courant, aucun théoricien, il tente de restituer les débats qui accompagnent chaque concept présenté ${ }^{1}$; 
- il s'efforce d'être lisible et accessible aussi bien pour des spécialistes d'autres disciplines que pour des non-spécialistes, dans une volonté délibérée de rompre avec un langage abscons qui a pu décrédibiliser certains travaux de didactiques ${ }^{2}$.

3 Nous remercions en tout cas nos collègues pour ces contributions, d'autant plus intéressantes qu'elles manifestent, au travers de la diversité des positions discursives adoptées, des questions et des critiques soulevées, ainsi que des références sollicitées, à quel point les discours des didacticiens sont tributaires de leur espace disciplinaire et à quel point les didactiques, au-delà de questions communes, fonctionnent différemment, contrairement donc aux assertions postulant leur uniformité sur le modèle de telle ou telle didactique, voire de telle ou telle théorie.

Nous souhaitons cependant ne pas en rester à ces premiers constats pour dire très clairement que nous considérons la posture adoptée par Yves Chevallard comme étrangère à nos conceptions de ce que doit être un débat scientifique, honnête et respectueux de l'autre. Ainsi l'ostension du laxisme critique est déjà pour le moins surprenante : «Examiner l'ouvrage dans son détail, ce qu'il mériterait, est impossible ici (nous espérons y revenir en un prochain travail). Un sondage express ferait-il l'affaire? " S'y ajoute une ironie constante qui nous paraît déplacée dans ce cadre, même s'il est facile d'y répondre. Ainsi, la critique d'« un certain engouement pour la notion allogène de Forme scolaire » et de ses conséquences supposées est stupéfiante sous la plume de celui qui a emprunté le concept de Transposition didactique au même socle sociologique. Ou encore, la référence à Wallon déconseillant à Zazzo l'usage de l'italique qui, selon lui, limiterait la liberté du lecteur, mériterait d'être méditée par Yves Chevallard lui-même quant à l'usage qu'il en effectue dans sa contribution. Comment encore, surtout dans ce contexte, interpréter l'expression qu'il choisit, le choix reutérien (les italiques sont d'Yves Chevallard), sinon comme une volonté de blesser en niant le travail collectif, sinon comme l'expression du fantasme de l'école de pensée et de l'impuissance à supposer une œuvre collective qui ne soit pas l'effet du choix d'un maître? S'ajoute à cela un imaginaire guerrier actualisé par un certain nombre de métaphores («l'histoire d'une guerre à partir des communiqués d'étatmajors seulement "; "l'effort de guerre »...). Nous considérons que cette manière belliciste d'envisager la vie scientifique a déjà causé beaucoup de tort à la didactique des mathématiques, voire aux autres didactiques. Que dire encore de ce long passage sidérant dans le cadre d'un débat scientifique - sur les guillemets? Nous pourrions nous contenter ici de remarquer qu'il suffit d'observer les écrits publiés pour se rendre compte qu'il n'a jamais existé « une » règle « classique ». Nous irons un peu plus loin. Il nous semble en effet que le contenu et la tonalité de ce passage, ainsi que le fantasme de la création d'une orthotypographie, relèvent d'un imaginaire de correcteur, d'une volonté de corriger l'autre. Nous abandonnerons donc ici Yves Chevallard à sa conception du débat.

5 Il est temps maintenant d'en venir aux critiques formulées par Jean-Paul Bernié et par Christian Orange qui nous semblent pouvoir être regroupées autour de trois axes : le choix des entrées retenues (et les oublis conséquents), les problèmes «internes » à certains articles, la conception (implicite/explicite) d'ensemble.

6 Tout dictionnaire repose de fait sur une sélection qui, en tant que telle, est discutable et mérite discussion. Dans cette perspective, Christian Orange analyse, à juste titre, la diversité des socles d'origine des concepts retenus et trace, sur cette base, un programme de recherche comparatiste auquel nous ne pouvons que souscrire. 
7 Précisons, à titre de contribution aux débats fondamentaux qu'il engage, que nous avons, après moult discussions, fondé notre sélection sur quatre catégories de concepts, qui ne recoupent pas totalement les siennes: les concepts reconnus dans différentes didactiques, qu'ils aient été forgés en leur sein ou repris d'autres disciplines (tels dévolution ou transposition didactique) ; les concepts, anciens ou plus récents, forgés dans un cadre didactique en réponse à des questions d'ordre didactique avec, provisoirement ou sur la durée, un champ d'extension plus restreint (niveau de formulation, conscience disciplinaire $\left.{ }^{3} . ..\right)$; les concepts, repris plus récemment d'autres disciplines de recherche et retravaillés dans une perspective didactique par différents courants au sein des didactiques; enfin des notions (telles enseignement, apprentissages, pédagogie...) dotées, selon nous, d'un statut paradoxal. En effet, elles sont employées constamment dans les articles ou les ouvrages des didacticiens et, de fait, semblent incontournables, tout en étant d'un usage très flou. Elles nous semblaient, par conséquent devoir nécessiter une formalisation théorique spécifique aux didactiques. Il s'agit là, sans nul doute d'un coup de force ... ou, du moins, d'une incitation à un travail collectif sur ces questions. C'est aussi, entre autres, ce qui fait pour nous de ce Dictionnaire non un aboutissement mais un outil heuristique. Il n'en demeure pas moins vrai que la liste retenue est, y compris pour nous, en mouvement, en fonction des critiques soulevées, en fonction des avancées des champs de recherche, en fonction de nos propres révisions quant aux choix adoptés... en fonction aussi de la nécessaire clarification collective à mener quant au lieu d'où parler des didactiques (cf. J.-P. Bernié) entre didactique(s), didactique générale et didactique comparée $e^{4}$.

8 Cela nous amène à nous arrêter sur les «entrées " dont nos collègues regrettent l'absence. Quatre principalement selon notre lecture mais qui soulèvent chacune, selon nous, des questions différentes appelant des réponses spécifiques, trop elliptiques sans doute en raison de l'espace dont nous disposons ce dont nous prions le lecteur de bien vouloir nous excuser. La notion de mésogenèse (qui figure bien comme élément d'une autre entrée) ne bénéficie effectivement pas d'une entrée autonome, ce qui a suscité de véritables discussions entre nous et sera, peut-être, modifié lors de la prochaine édition du Dictionnaire. La dimension « quasi trinitaire du triplet » évoqué par Jean-Paul Bernié nous a en effet moins frappés que lui (voir les usages largement autonomisés de chronogenèse et de topogenèse) ainsi que la valeur heuristique de ce concept qui nous a paru moindre, comme nous ont semblé plus discrets ses usages en dehors de l'espace de la didactique des mathématiques ${ }^{5}$. Nous sommes en revanche entièrement d'accord avec lui pour penser qu'il est nécessaire d'introduire une entrée interactions didactiques ${ }^{6}$, d'ores et déjà prévue pour la prochaine édition, en signalant néanmoins - et c'est pour partie une explication à ce décalage temporel - qu'il s'agit tout à la fois d'une notion qui nous paraît à la fois largement utilisée et indispensable tout en étant insuffisamment construite en tant que concept.

9 Pour ce qui concerne le concept de zone de proche développement, nous pensons - mais cela relève de la question des cadres théoriques sur laquelle nous reviendrons un peu plus loin - qu'il s'agit d'un concept sans nul doute fondamental pour certaines questions jugées primordiales par certains courants au sein des didactiques ${ }^{7}$, comme ceux d'habitus ou d'historicité peuvent l'être pour d'autres, mais qui n'est ni un concept appartenant aux didactiques, ni un concept qui répond aux questions centrales des didactiques, ni un concept retravaillé dans le cadre des didactiques. Dès lors, il ne nous semblait pas devoir/pouvoir être retenu et, si tel avait été le cas, nous ne voyions plus 
de limites à ce dictionnaire qui aurait été tenu d'intégrer nombre de concepts issus des principales disciplines contributoires. Reste la possibilité d'une entrée "problème " soulevée par les remarques de Christian Orange. Nous n'avons pas, après bien des discussions, de position tranchée. Peut-être penchons-nous plutôt, à l'heure actuelle, et cela ne nous semble pas contradictoire avec ses critiques, vers une accentuation de certaines formes de problématisation de nos entrées (nous y reviendrons aussi dans la suite de ce texte).

maintenant à la seconde catégorie de critiques, critiques concernant la manière de traiter telle ou telle entrée (en remarquant cependant qu'aucun dictionnaire ne saurait échapper à ce genre de critiques). Nous enregistrons ainsi les remarques de Jean-Paul Bernié sur "contexte/milieu», tout en n'étant pas sûrs de vouloir aller tout à fait dans son sens, au détriment, par exemple, des questions d'ordre socio-culturel. Nous enregistrons ${ }^{8}$ aussi ses critiques sur " métalangage » et " activités métalinguistiques» tout en signalant que certes nous n'avons pas donné la prééminence aux conceptions qu'il défend ${ }^{9}$ mais qu'en revanche nous avons replacé ces conceptions au sein de débats qui s'interrogent, d'une part sur les formes scolaires et non scolaires du/des métalangage(s) et des activités métalinguistiques, d'autre part sur le rapport entre ces formes et ces usages propres à l'école avec des dispositions socialement construites, potentiellement génératrices d'effets d'inégale réussite (voir, sur ce point, les travaux de Bernard Lahire). Et, s'il est vrai que la définition fournie à l'entrée "Outils» peut paraître, comme toute définition ${ }^{10}$, restrictive, elle n'est cependant pas réduite à « aides didactiques ", puisqu'il est bien précisé dans le passage mentionné que «la notion d'outil permet[te] ici d'englober les aides didactiques, mais également d'autres éléments ». En revanche, il nous a paru important, pour cette entrée, de porter l'accent sur la description nécessaire de ces outils, encore insuffisamment effectuée à notre sens, ainsi que sur leur dimension didactique qui ne saurait, selon nous, se confondre avec leur fonctionnement psychologique supposé. Pour ce qui est de l'article "Concepts-champs conceptuels", nous ne pouvons qu'être d'accord avec Christian Orange lorsqu'il remarque qu'une approche catégorielle de type sémantique a pris le pas sur une approche épistémologique. Nous reviendrons, en revanche, sur la conception d'ensemble de l'écriture des articles du Dictionnaire qui se trouve, elle aussi, interrogée.

11 Nous menons - particulièrement en didactique du français et depuis de longues années - des débats que nous jugeons importants autour du concept de discipline avec nos collègues genevois ou bordelais. C'est dire que nous ne saurions réduire ce débat à des réponses cavalières. Notons seulement ici, en tant que jalons pour des discussions futures, que nous ne pensons pas que l'élève soit absent des développements de cette entrée (voir ce qui concerne le point de vue, les visées, les pratiques, les relations aux espaces extrascolaires, la conscience disciplinaire...), même s'il est vrai (point de débat récurrent avec Jean-Paul Bernié) que nous ne voyons pas pourquoi nous aurions privilégié la dimension psychologique au détriment des autres dimensions. Quant à la notion de "discipline formelle", reprise d'Herbart, elle nous pose de multiples problèmes, entre autres, celui de savoir ce que pourrait être une discipline non formelle au sein de la forme scolaire.

Demeure le problème de la thématisation de la question du/des savoir(s) dans le Dictionnaire, soulevée aussi bien par Jean-Paul Bernié que par Christian Orange. D'un côté, nous assumons le choix d'intégrer savoirs dans l'entrée "Contenus 
d'enseignement et d'apprentissages » (et dans bien d'autres), cela afin de rompre avec le fétichisme attaché à la notion de savoirs par certains didacticiens, fétichisme qui fait qu'on n'interroge pas suffisamment cette notion, qu'on néglige le fait qu'on n'enseigne pas que et qu'on n'apprend pas que des savoirs au sein des disciplines, fétichisme qui renvoie peut-être aussi au statut ininterrogé des savoirs au sein de certaines didactiques pour qui les savoirs de leur discipline (scolaire/universitaire/de recherche?) seraient plus "scientifiques» que ceux d'autres disciplines. Complémentairement, nous ne pouvons souscrire à l'idée que les savoirs scolaires auraient, en quelque sorte en soi, «le pouvoir de susciter du développement». Cela nous semble idéaliste ${ }^{11}$ à la fois en raison de l'abstraction des savoirs scolaires des pratiques qui les actualisent et au regard des effets (différenciés) sur les élèves. En revanche, nous ne pouvons que souscrire à quatre remarques critiques: la typologie initiale repose encore trop sur des catégories psychologiques; le débat autour de la notion de compétences est insuffisant; la question des "éducations à " n'est pas suffisamment construite (elle devrait faire, elle aussi, l'objet d'une entrée spécifique dans la prochaine édition); et surtout, c'est absolument juste, les questions du statut, de la nature, voire de l'histoire, des savoirs scolaires sont insuffisamment développées. Cela doit-il faire l'objet d'une entrée autonome, être plus explicitement thématisé dans l'entrée «Contenus... » ou être expansé dans les diverses entrées du Dictionnaire? Nous ne le savons pas encore.

Venons-en maintenant, trop brièvement encore sans doute, aux discussions sur la conception d'ensemble de ce Dictionnaire. Il est certain, comme le remarque Chevallard, que l'analyse génétique (les italiques sont de lui) est peu développée mais il n'est pas sans savoir qu'une archéologie des concepts, pour être sérieuse, aurait nécessité plusieurs ouvrages. Nous n'ignorons nullement les cadres de pensée de Bernié, de Chevallard ou de bien d'autres, mais nous avons effectué le choix, effectivement discutable, de ne pas consacrer d'entrée aux théories pour deux raisons principales :

- d'une part, la notion même de théorie nous semblait soulever nombre de problèmes épineux (ainsi certains parlent de "théorie de la transposition didactique » pour ce qui nous paraît relever d'un concept; ou encore faut-il considérer comme tel un paradigme de questions, par exemple, la « didactique curriculaire»?);

- d'autre part, dans certains cas, il nous semble que cela interroge le statut même (l'autonomie notamment) des didactiques lorsqu'il est question de psychologie, d'anthropologie ou de sociologie (cf. la sociodidactique) ; s'il n'existe certes pas (mais qui le prétend ?) de «Grande Muraille », la question se pose cependant de clarifier le statut de ces "théories». S'agit-il d'intégrer les didactiques comme sous-continents d'autres disciplines de recherche ou s'agit-il de sous-continents des didactiques? De fait, la discussion nous semble ouverte et chacune de ces « théories » a, sans nul doute, à clarifier sa position.

Nous sommes, en tout cas, sensibles à deux critiques importantes soulevées par Christian Orange. La première concerne le caractère insuffisamment problématisé, selon lui, de nos entrées. Dont acte. Sachant que cette problématisation était pourtant fortement inscrite dans notre projet, ainsi qu'en attestent pour chaque entrée les rubriques "Éléments d'éclairage » et "Problèmes, questions, débats ", somme toute assez rares dans de tels outils. La seconde critique nous paraît particulièrement importante au regard du champ des didactiques: nous n'aurions pas suffisamment référé les concepts présentés aux domaines de savoirs, aux contenus d'enseignement et d'apprentissages qui constituent leur socle originel. Dont acte ici encore, malgré cette 
volonté de référence présente dans notre projet. Sur ces deux points, entre autres, il nous semble clair que le Dictionnaire peut et doit être amélioré dans une prochaine édition.

Reste encore à débattre de l'idée, explicitement ou implicitement exprimée par nos collègues mais en tout cas récurrente, selon laquelle il faudrait nécessairement qu'il y ait un modèle unitaire qui soit au fondement de ce Dictionnaire et qui le structure. Nous répondrons ici que tel n'est absolument pas le cas. D'une part, parce qu'il nous semble que c'est radicalement opposé à l'idée même d'un Dictionnaire tentant de rendre compte, sans a priori, d'un champ disciplinaire. Ce serait, de surcroît absurde, au regard de la diversité des concepts issus d'époques, de didactiques, de courants, d'auteurs... différents. D'autre part, parce que l'idée même d'un modèle unique susceptible de permettre d'appréhender l'intégralité d'un champ de recherche mérite d'être interrogée. Enfin, parce que s'il est vrai que nous (i.e. les auteurs de ce Dictionnaire) avons une culture commune et produisons un ou plusieurs point(s) de vue via cet ouvrage, il est vrai aussi que nous n'avons pas de modèle commun unique ${ }^{12}$. Il suffit de lire nos travaux pour s'en convaincre. Au-delà, et c'est peut-être ce qui nous unit, c'est sans doute un principe fort pour nous que de refuser dogmes, maitres à penser et épigones...

Il nous reste à remercier encore nos collègues pour leur lecture attentive, pour leurs remarques et pour cette discussion qui nous a permis, au moins en partie, de clarifier nos positions et d'envisager, de manière mieux outillée, la prochaine édition de ce Dictionnaire.

\section{NOTES}

1. Nous souhaitions que cet ouvrage fût véritablement fondé sur le débat: par la confrontation de concepts d'origines diverses, par la mise en débat accompagnant chacun de ces concepts.

2. Autant dire que nous ne partageons pas la conception de l'écriture que défend ici Yves Chevallard. D'une part, parce qu'il nous paraît paradoxal de vouloir réaliser un outil destiné à aider à entrer dans un domaine de connaissance en rendant les définitions "assez incompréhensible[s]»; d'autre part, parce que la simplicité d'une définition est toute relative et plutôt le point de départ d'un travail d'appropriation (sur ce qui la fonde, sur ce qu'elle permet...) qu'un point d'arrivée.

3. Notion introduite en 2003 (et non en 2007) et construite à partir d'un retravail de recherches et de notions utilisées notamment en didactiques de l'histoire et géographie, des sciences ...

4. La liste des concepts retenus, en fonction de la catégorisation signalée, obéit in fine, à la volonté de sélectionner les concepts qui ont fondé aussi bien les didactiques que les discussions entre elles; c'est ce qui en fait, à nos yeux, des concepts fondamentaux. 
5. Nous avons préféré présenter le concept de milieu en posant les termes, tout du moins nous l'espérons, d'une confrontation entre les deux concepts de milieu et contexte.

6. Et, sans doute, une entrée " Relation didactique ».

7. Et, qui, de fait, interrogent tous les courants. Mais nous persistons à penser que, par exemple, la question des relations entre enseignement, apprentissages et développement, si elle est susceptible d'interroger les didactiques n'est cependant pas centrale pour elles qui, d'ailleurs, pour beaucoup de courants et de recherches, s'en passent fort bien.

8. Cela signifie, en l'occurrence, que nous prenons au sérieux ses remarques mais que nous avons besoin de plus de temps pour y réfléchir et construire une position commune.

9. Tout en ne sachant bien ni ce que serait une "orthodoxie vygotskienne », surtout dans le champ des didactiques, ni ce que recouvre l'expression "didactiques sèches " au regard, par exemple, de l'histoire de la didactique du français, de celle des sciences, et de bien d'autres encore...

10. C'est bien pour cette raison que nous avons appelé cette partie, non pas " Définition », mais « Première approche de la/les notion(s) ».

11. D'une certaine manière, nous nous sentons plus proches ici des positions de Goody lorsqu'il critique l'idéalisme de Vygotski (voir notamment J. Goody: Entre l'oralité et l'écriture, 1993, trad. fr., PUF, 1994).

12. Il en est de même au sein du laboratoire Théodile-CIREL.

\section{AUTEURS}

\section{YVES REUTER}

(université Lille 3, Théodile)

BERTRAND DAUNAY

(université Lille 3, Théodile)

ISABELLE DELCAMBRE

(université Lille 3, Théodile)

CORA COHEN-AZRIA

(université Lille 3, Théodile)

DOMINIQUE LAHANIER-REUTER

(université Lille 3, Théodile) 